\title{
Cone quality and seed efficiency in the clones from mutational witches brooms of Pinus sibirica
}

\author{
Olga Polyakova ${ }^{1,2, *}$, Evgeniya Zhuk $^{1}$, and Sergei Goroshkevich ${ }^{1}$ \\ ${ }^{1}$ Institute of Monitoring of Climatic and Ecological Systems, Russian Academy of Sciences, Siberian \\ Branch 634055 Tomsk, 10/3 Academichesky ave., Russia \\ ${ }^{2}$ Tomsk State University, 634050 Tomsk, 36 Lenina ave., Russia
}

\begin{abstract}
Mutational witches' broom is a fragment of the tree crown with abnormal morphogenesis including slow shoot growth, abundant branching and decreased apical dominance which caused by somatic mutation that presumably originates in the apical meristem bud. To study the variability in the cone quality and seed efficiency in witches' broom clones of Pinus sibirica, the cone size and structure were assessed in 21 mutant clones. The mutant clones were significantly differentiated both by the cone traits and seed quality. Their cone size varied from less than 3 to $4 \mathrm{~cm}$. The clones had large number of sterile scales in proximal and distal cone parts, and from 16 to 39 fertile scales per cone. Cones from all mutant clones were inferior to average normal cones of the species. Six clones had large portion of filled seeds, more than $70 \%$, and the other clones had less than $50 \%$ of filled seeds. The clones had $10-33 \%$ of empty seeds and $1-73 \%$ of seeds with collapsed megagametophyte per cone. In general, the seed quality in mutants was quite low, but individual clones had almost normal seed quality, and their seed efficiency was within the species norm.
\end{abstract}

\section{Introduction}

Mutational witches' broom (WB) is a fragment of the tree crown with abnormal morphogenesis including slow shoot growth, abundant branching and decreased apical dominance A somatic mutation that presumably originates in the bud in the apical meristem is considered as a cause of the WB [1]. Unlike pathological WB, mutational WB are sporadically distributed and have a healthy appearance, normal reproduction, and normal longevity [2-3]. Mutational WB is a major source of the majority of ornamental dwarf conifer cultivars [4]. To date, certain patterns in the morphological traits variability of WB clones were revealed [5]. Although the seed progeny involvement in breeding process has great importance, the information concerning the variability of cone structure and seed efficiency in mutant clones is scarce. The objective of the work was to study the variability of the cone quality and seed efficiency in WB clones of Pinus sibirica.

\footnotetext{
* Corresponding author: polyakova_olga93@mail.ru
} 


\section{Materials and Methods}

\subsection{Plant material}

The investigation was carried out on grafted WB clones of Pinus sibirica. The experiment was conducted at the Kedr Field Station, which is managed by the Institute of Monitoring of Climatic and Ecological Systems, Russian Academy of Sciences, Siberian Branch $\left(56^{\circ} 13^{\prime} \mathrm{N} 84^{\circ} 51^{\prime} \mathrm{E}, 78 \mathrm{~m}\right.$ above sea level, south-east of West Siberian Plain, southern taiga). Plant material for grafting were 21 trees with morphologically different mutational WB. Parental WB had different crown density, which was defined by the combination of three main traits, abundance of branching, shoot length and degree of apical dominance. The more abundant branching, shorter shoots and lower apical dominance, the WB looks denser.

In 2007, scions from each WB and NC were grafted onto local five-year-old Siberian stone pine rootstocks. The grafted plants were grown with $1 \times 0.5 \mathrm{~m}$ spacing in rows, each clone contained 10-20 ramets. At the clone age of 10 years, cones from all cone-bearing ramets were collected. If a clone had weak cone-bearing, a whole cone crop (3-9 cones) was taken for analysis. If a clone had abundant cone-bearing, 15 cones from the whole crop were randomly taken for analysis.

\subsection{Measurements and data analysis}

The following traits were measured in the cones: cone length, cone diameter, number of sterile scales in proximal cone part, number of fertile and sterile scales in medial cone part, number of sterile scales in distal cone part, number of rudimentary and full-grown seeds. Number of unpollinated ovules was calculated as doubled number of sterile scales in medial cone part. Among full-grown seeds the number of filled and empty seeds, and also number seeds with collapsed megagametophyte were determined using X-ray analysis. Seed efficiency was calculated as portion of filled seeds from the total number of ovules. Normality was tested using the Kolmogorov-Smirnov test. If the distribution was normal, ANOVA and Newman-Keuls test were used to compare clones. In the other cases, the Kruskal-Wallis and non-parametric Dunn's multiple comparison tests were used.

\section{Results}

All cone traits were highly variable in WB clones. Fifteen percent of clones had very small cones with length and diameter less than $3 \mathrm{~cm}$. Twenty percent of clones had quite big cones compared with other clones, their length were more than $4 \mathrm{~cm}$ and diameter were more than $4.5 \mathrm{~cm}$. The other clones had intermediate cone size. All the clones had large number of sterile scales in proximal and distal cone parts (Fig. 1). In some clones more than half of total number of cone scales were sterile scales. The number of fertile scales in medial cone part was even more differentiated among clones than cone size, from 16 to 39 fertile scales per cone.

All the clones had almost equally high number of unpollinated ovules in medial cone part, about $25 \%$ (Fig. 2). In general, number of rudimentary seeds was quite low with some variations among the clones. Number of full-grown seeds varied significantly among the clones, from 6-11 to 36-42 full-grown seeds per cone. Seed efficiency was from 5 to $48 \%$ in different clones and $27 \%$ in average. 


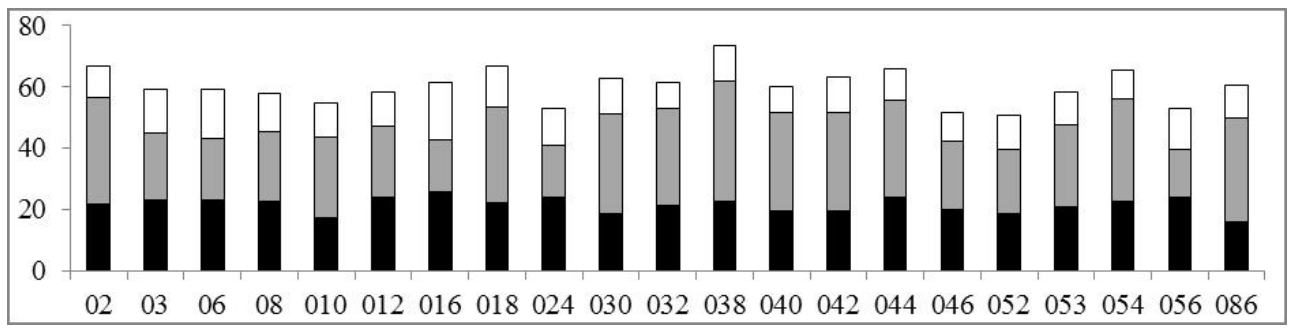

Fig. 1. Number of cone scales in mutant clones: sterile scales in proximal cone part (black), fertile scales in medial cone part (gray), sterile scales in distal cone part (white)

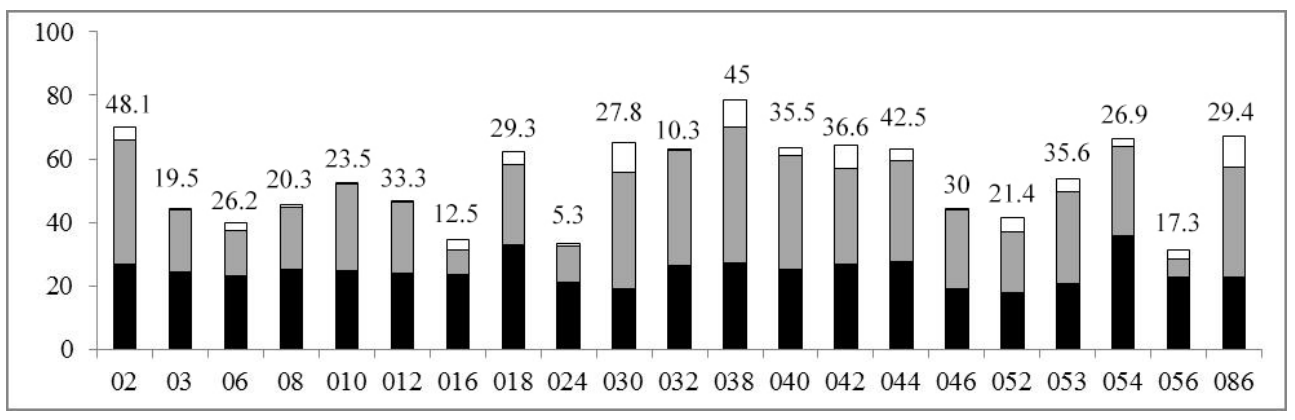

Fig. 2. Number of different types of seeds in mutant clones: unpollinated ovules (black), full-grown seeds (gray), rudimentary seeds (white). The values above the columns are seed efficiency (\%)

The quality of full-grown seeds also significantly varied among the clones. Six clones had large portion of filled seeds (more than 70\%), half of clones had medium portion of filled seeds (about 50\%), and the other clones had low portion of filled seeds (less than $40 \%$ ) (Fig. 3). All the clones had about $20 \%$ of empty seeds, except 2 clones having much more empty seeds. Portion of seeds with collapsed megagametophyte significantly varied among the clones. In more than half of clones their portion was moderate, but several clones had $30-70 \%$ of these seeds.

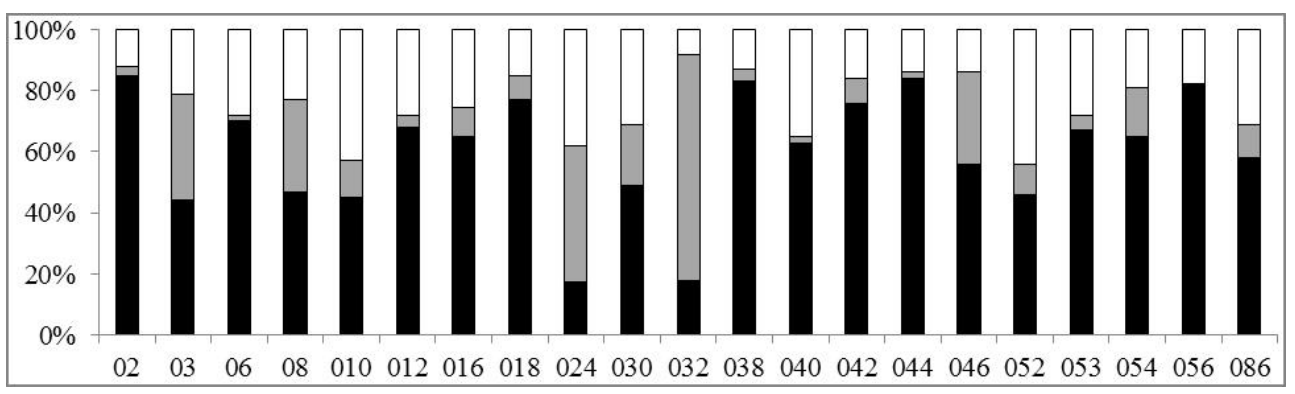

Fig. 3. Quality of full-grown seeds in mutant clones: portion of filled seeds (black), seeds with collapsed megagametophyte (gray) and empty seeds (white)

\section{Discussion}

Mutational WB cones are generally smaller than normal cones in some species. This pattern is typical both for soft pines, such as P. strobus [3], and for hard pines, such as P. sylvestris [6], P. banksiana [7] and P. halepensis [8]. Usually WB cones were 1.2-2-fold smaller, however, in some cases WB cones did not differ from normal cones [9]. Average cone length in $P$. sibirica growing in wild forest is $7-8 \mathrm{~cm} \mathrm{[10].} \mathrm{The} \mathrm{mutant} \mathrm{clones} \mathrm{had} \mathrm{2-fold}$ 
smaller cone size, and there were no clones with cone size at least approximately close to normal species cones.

Number of cone scales is closely related to the cone size. The mutant clones with the smallest cones had the smallest number of scales. It is possible that the cone size and the specific cone structure with large portion of sterile scales are related to the specific WB crown structure [5]. Large portion of unpollinated ovules could be caused by abnormal density of WB crown and associated pollination problems.

Quality of full-grown seeds were not related to the cone size. A clone with typical WB cones, that were good enough visually, had largest portion of seeds with collapsed megagametophyte. Among Pinus sibirica trees there are also ones producing cones with low quality seeds [11], therefore, it was impossible to determine whether low seed quality is WB mutations attribute or it depend on parental tree features. In general, WB clones of had low seed quality, however, individual clones had almost normal seed quality, and their seed efficiency was within the species norm.

This work is supported by the Russian Science Foundation (grant no. 18-16-00058).

\section{References}

1. J. Duffield, J. Wheat, Silvae Genet 12, 129-133 (1963)

2. D.C. Buckland, J. Kuijt, For. Sci. 3, 236-242 (1957)

3. A.J. Fordham, Arnoldia 24, 29-50 (1967)

4. A. Farjon, A handbook of the world's conifer (Brill, Leiden-Boston, Netherlands, 2017)

5. E. Zhuk, G. Vasilyeva, S. Goroshkevich, Trees 29, 107+-1090 (2015)

6. V.V. Shul'ga, Lesovedenie 3, 82-86 (1979)

7. T.D. Rudolph, D.M. O'Malley, E.A. Reed, Proceedings of the North Central Tree Improvement Conference, 162-174 (1983)

8. P. Vrgoc, Proceedings of XX International Eucarpia Symposium, Section Ornamentals, Strategies for New Ornamentals, 199-205 (2002)

9. V.I. Noskov, S.F. Negrutzkiy, Sci. Notes of Voronezh For. Engineering Institute 15, 207-211 (1956)

10. V.N. Vorobyev, Biological basis of complex use of stone pine forests (Novosibirsk, Nauka, 1983)

11. E.V. Titov, Kedr. The king of the Siberian taiga (Moscow, Kolos, 2007) 